\title{
Não-adesão à terapêutica medicamentosa do glaucoma
}

\author{
Noncompliance with drug therapy for glaucoma
}

\author{
Aldemar Nemesio Brandão Vilela de Castro ${ }^{1}$ \\ Wander Araújo Mesquita ${ }^{2}$
}

Trabalho realizado na Clínica Oftalmológica do Hospital Israel Pinheiro (HGIP), Instituto de Previdência dos Servidores de Minas Gerais - IPSEMG - Belo Horizonte (MG) - Brasil.

${ }^{1}$ Doutor. Coordenador da Clínica Oftalmológica do Hospital Israel Pinheiro - HGIP, Instituto de Previdência dos Servidores de Minas Gerais - IPSEMG - Belo Horizonte (MG) - Brasil.

${ }^{2}$ Farmacêutico-bioquímico. Mestre em Ciências da Saúde pelo IPSEMG - Belo Horizonte (MG) - Brasil.

Endereço para correspondência: Wander Araújo Mesquita. Rua Afrânio Peixoto, 2.211 - Apto. 102-E - Divinópolis (MG) CEP 35501-284

E-mail: wanderfarmaceutico@bol.com.br

Recebido para publicação em 24.04.2007

Última versão recebida em 21.08.2007

Aprovação em 28.08.2007

\begin{tabular}{l} 
RESUMO \\
\hline Introdução: O tratamento do glaucoma é feito inicialmente por meio do uso \\
de colírios, com a finalidade de diminuir a pressão intra-oculare minimizar os \\
danos ao nervo óptico. A falta de adesão à terapia medicamentosa pode ser \\
influenciada por fatores relacionados aos pacientes, aos médicos e aos \\
medicamentos. Objetivos: Estimar a taxa de não-adesão ao tratamento com \\
colírios antiglaucomatosos e verificar a interveniência de possíveis fatores \\
para a não-adesão. Métodos: Foi realizado um estudo do tipo transversal, na \\
Clínica Oftalmológica do Hospital Governador Israel Pinheiro, em Belo \\
Horizonte, Minas Gerais. Foram aplicados questionários a 102 pacientes \\
glaucomatosos para a coleta de informações a respeito do nível de não- \\
adesão e da interveniência de possíveis fatores. Resultados: A taxa de não- \\
adesão ao tratamento medicamentoso do glaucoma foi de 21,5\%. Os fatores: \\
deixar de usar os colírios por falta de dinheiro (p=0,035) e realização de \\
cirurgia corretiva (p=0,049), contribuíram de forma significativa para a não- \\
adesão. Conclusão: Mais de um quinto dos pacientes glaucomatosos não \\
seguiram o tratamento medicamentoso com colírios de forma adequada. \\
Doação de colírios a todos os pacientes glaucomatosos de condições \\
econômicas desfavoráveis, ações governamentais para diminuição dos \\
preços dos colírios, informações sobre a continuidade do uso dos colírios \\
após a realização de cirurgias corretivas, ajudariama diminuir as taxas denão- \\
adesão.
\end{tabular}

Descritores: Glaucoma/quimioterapia; Cooperação do paciente; Recusa do paciente ao tratamento; Soluções oftálmicas

\section{INTRODUÇÃO}

A adesão à terapêutica medicamentosa é comumente utilizada para descrever a habilidade do paciente em usar uma medicação conforme orientações prestadas por profissionais da saúde ${ }^{(1)}$. Dados da literatura mostram que existem falhas no seguimento de regimes terapêuticos medicamentosos, principalmente em relação às doenças crônicas ${ }^{(2)}$.

O glaucoma é uma doença crônica, silenciosa, caracterizada pela perda gradual do campo de visão e por dano ao nervo óptico, sendo considerada um problema de saúde pública, visto que a principal conseqüência imposta por esta patologia é a perda de visão irreversível ${ }^{(3-5)}$. Estima-se que em 2020, o mundo terá 1,2 bilhões de glaucomatosos com idade acima de $60 \operatorname{anos}^{(6)}$. No Brasil, apesar das dificuldades em obtenção de dados, estima-se que 500 mil brasileiros com mais de 40 anos sejam portadores de glaucoma $^{(7)}$.

O tratamento do glaucoma é feito inicialmente através do uso de colírios, com a finalidade de diminuir a pressão intra-ocular $(\mathrm{PIO})^{(8-9)}$ e minimizar os danos ao nervo óptico. A falta de adesão à terapia medicamentosa do glaucoma pode ser influenciada por fatores relacionados aos pacientes, aos médicos e aos medicamentos ${ }^{(10)}$. 
O presente artigo tem como objetivos principais:

a) Estimar a taxa de não-adesão ao tratamento com colírios antiglaucomatosos;

b) Verificar a interveniência de possíveis fatores para a não-adesão à terapêutica medicamentosa do glaucoma.

\section{MÉTODOS}

Foi realizado um estudo do tipo transversal, na Clínica Oftalmológica do Hospital Governador Israel Pinheiro (HGIP), em Belo Horizonte, Minas Gerais. A pesquisa foi iniciada em agosto de 2006, após aprovação pelo Comitê de Ética da instituição, sendo finalizada em novembro do mesmo ano.

Foram aplicados questionários a 102 pacientes glaucomatosos para a coleta de informações a respeito do nível de nãoadesão, sendo considerados não-aderentes aqueles que deixavam de pingar duas ou mais doses do(s) colírio(s) por semana. Além disso, foram obtidos dados relacionados a possíveis fatores intervenientes a não-adesão à terapêutica medicamentosa do glaucoma: sexo, idade, raça, número de colírios utilizados, número de aplicações diárias, renda familiar mensal, gasto mensal com os colírios, nível de escolaridade, efeitos adversos, realização de cirurgia corretiva, dificuldade em pingar os colírios, consultas com oftalmologista, doenças coexistentes, e deixar de usar os colírios por falta de dinheiro. A coleta de dados foi realizada com pacientes que estavam em espera por consulta oftalmológica e que preenchiam os critérios de inclusão. A aplicação dos questionários foi feita por profissionais previamente treinados, os quais antes de iniciarem as entrevistas, apresentavam aos pacientes os termos de esclarecimento sobre a pesquisa e estando estes de acordo em participar, assinavam termo de consentimento autorizando a realização da mesma. O modelo de questionário utilizado era constituído quase que na sua totalidade por questões fechadas do tipo múltipla escolha, conforme modelo em anexo. As perguntas eram feitas aos pacientes da mesma forma como descrito no questionário, ficando os aplicadores responsáveis pela anotação das respostas.

Foram adotados os seguintes critérios para inclusão de pacientes:

a) diagnóstico de glaucoma confirmado;

b) maiores de 18 anos;

c) em uso de colírios antiglaucomatosos;

d) podendo ter realizado ou não cirurgia corretiva de glaucoma.

Os dados levantados foram analisados estatisticamente pelo teste qui-quadrado com nível de significância de 5\%, através do programa Epi Info versão 3.2.2.

\section{RESULTADOS}

A taxa de não-adesão ao tratamento medicamentoso do glaucoma foi de $21,5 \%$. Os pacientes apresentaram idade igual a $63,5 \pm 11,45$ anos, com predominância do sexo feminino (72,5\%), sendo a amostra constituída de 38,2\% de indivíduos brancos, $36,3 \%$ de pardos, $17,6 \%$ de negros e $0,95 \%$ de outros. Em relação à terapêutica medicamentosa, 24 (23,5\%) pacientes necessitavam de auxílio para instilar os colírios, 16 (15,7\%) pacientes recebiam ajuda e/ou doação para aquisição dos medicamentos antiglaucomatosos (Tabela 1). A instilação de colírios em horários inadequados foi relatada por 11 (10,7\%) dos pacientes e situações de esquecimento do uso de colírios foram relatadas por $12(11,7 \%)$ dos pacientes (dados não analisados).

$\mathrm{Na}$ amostra em estudo, 33 (32,3\%) dos pacientes sofriam de hipertensão, 18 (17,6\%) sofriam de hipertensão e diabetes, 5 $(4,8 \%)$ sofriam de diabetes (Tabela 2$)$.

O maleato de timolol foi o colírio mais utilizado pelos pacientes $(49 \%)$, seguido de tartarato de brimonidina $(23,5 \%)$, bimatoprost (20,6\%), travaprost $(16,7 \%)$, latanoprost $(11,7 \%)$, dorzolamida + maleato de timolol $(9,8 \%)$, brimonidina + maleato de timolol $(6,8 \%)$ e betaxolol (4,8\%) (Figura 1).

Os efeitos adversos mais notados pelos pacientes foram ardência $(45,1 \%)$, gosto amargo na boca $(20,6 \%)$, irritação ocular $(17,6 \%)$, lacrimejamento $(11,7 \%)$, turvação visual $(9,8 \%)$ e cefaléia $(9,8 \%)$ (Figura 2$)$.

Dentre os fatores levantados como possíveis intervenientes a não-adesão, sexo $(\mathrm{p}=0,575)$, idade $(\mathrm{p}=0,079)$, raça $(p=0,559)$, efeitos adversos $(p=0,39)$, doenças coexistentes $(\mathrm{p}=0,849)$, número máximo de instilações por dia $(\mathrm{p}=0,149)$, número de colírios utilizados $(\mathrm{p}=0,393)$, dificuldade em pingar os colírios $(\mathrm{p}=0,228)$, nível de escolaridade $(\mathrm{p}=0,377)$, renda familiar ( $\mathrm{p}=0,748)$, gasto mensal $(\mathrm{p}=0,198)$ e consultas com oftalmologista $(\mathrm{p}=0,083)$ não foram estatisticamente associados à não-adesão. De forma contrária, os fatores: deixar de usar os colírios por falta de dinheiro $(\mathrm{p}=0,035)$ e realização de cirurgia corretiva ( $\mathrm{p}=0,049)$, contribuíram de forma estatisticamente significativa para não-adesão ao tratamento antiglaucomatoso (Tabela 3). Nota-se que para execução dos cálculos estatísticos relacionados a esta tabela, foram extraídos dados amostrais, referentes aos fatores: raça, doenças coexistentes, realização de cirurgia corretiva, nível de escolaridade, renda familiar, gasto mensal e deixar de usar os colírios por falta de dinheiro. Isto ocorreu porque alguns pacientes não souberam ou não quiseram responder às perguntas realizadas, e também pelo fato das respostas dadas não se enquadrarem ao questionário.

\section{DISCUSS ÃO}

A taxa de não-adesão encontrada foi de 21,5\%. Estu$\operatorname{dos}^{(3,11-12)}$ que semelhantemente ao nosso, adotaram a entrevista como metodologia de pesquisa e a perda de mais de uma dose de colírio por semana como conceito de não-adesão, relataram taxas de não-adesão variando entre $12,8 \%^{(11)}$ e 27,5\% ${ }^{(3)}$. Em outros estudos ${ }^{(8,10,13-20)}$ onde os conceitos de não-adesão e as metodologias aplicadas foram variáveis e/ou não definidas, a taxa de não-adesão variou de $4,6 \%^{(10)}$ a $59 \%{ }^{(20)}$. Um destes ${ }^{(13)}$ mostrou também que a taxa de não-adesão conseguida através de entrevistas, era subestimada, em torno de $21 \%$, quando comparada às taxas mensuradas por monitor. 
Anexo

Questionário - Dissertação de mestrado em Ciências da Saúde: Fatores para não-adesão à terapêutica medicamentosa do glaucoma

Paciente:

Sexo: ( ) M ( ) F Idade: anos

Prontuário : Grupo racial: ( ) Negro ( ) Branco ( ) Pardo ( ) Asiático ( ) Outro

Diagnóstico:

Idade quando foi detectado o glaucoma? anos.

O glaucoma foi diagnosticado em consulta de rotina com oftalmologista? ( ) sim ( ) não

Já passou por cirurgia de glaucoma? ( ) sim ( ) não

Se sim, quando?

\section{Tratamento:}

O tratamento medicamentoso foi iniciado logo após o diagnóstico do glaucoma? ( ) sim ( ) não

Qual(is) o(s) colírio(s) você está utilizando atualmente no tratamento do glaucoma e quantas aplicações são feitas por dia?

Colírios utilizados

( ) Maleato de timolol/Timoptol ${ }^{\circledR}$ Posologia ( $\mathrm{n}^{\circ}$ de aplicações por dia)

( ) Acetazolamida/Diamox ${ }^{\circledR}$

( ) Cloridrato de pilocarpina/Isopto Carpine ${ }^{\circledR}$

( ) Latanoprost/Xalatan ${ }^{\circledR}$

( ) Dorzolamida/Trusopt ${ }^{\circledR}$

( ) Betaxolol/Betoptic ${ }^{\circledR}$

( ) Dorzolamida + maleato de timolol/Cosopt ${ }^{\circledR}$

( ) $1($ ) $2($ ) 3

( ) $1($ ) 2() 3

( ) $1($ ) $2($ ) 3

( ) $1($ ) $2($ ) 3

( ) $1($ ) $2($ ) 3

( ) $1($ ) $2($ ) 3

( ) Latanoprost + maleato de timolo/Xalacom ${ }^{\circledR}$

( ) $1($ ) $2($ ) 3

( ) Tartarato de brimonidina/Alphagan ${ }^{\circledR}$

( ) Tartarato de brimonidina + maleato de timolol/Combigan $^{\circledR}$

( ) $1($ ) $2($ ) 3

( ) $1($ ) $2($ ) 3

( ) Bimatoprost/Lumigan ${ }^{\circledR}$

( ) Travoprost/Travatan ${ }^{\circledR}$

( ) Outro

( ) $1($ ) $2($ ) 3

( ) $1($ ) $2($ ) 3

( ) $1($ ) 2() 3

( ) $1($ ) $2($ ) 3

A posologia é seguida à risca? ( ) sim ( ) não

Se não, por que?

Você sente alguma reação adversa? ( ) sim ( ) não

Se sim, qual(is)?

( ) Irritação ocular ( ) Ardência （）Cefaléia （）Turvação visual （）Perda de peso （）Gosto amargo na boca

( ) Ressecamento da conjuntiva ( ) Lacrimejamento ( ) Hipotensão ( ) Outra

Em média quantas vezes você deixa de pingar o colírio em uma semana? ( ) uma （） duas （） três ( ) mais que três Sempre pinga o número de gotas prescrito pelo médico? ( ) sim ( ) não

Se não, por que?

Você acha se pingar mais gotas, o glaucoma melhora mais depressa? （） sim （）não ( ) não tem idéia

Você mesmo pinga o colírio? ( ) sim ( ) não

Se não, quem pinga? ( ) filho(s) ( ) esposa ( ) marido ( ) pai ( ) mãe ( ) outro

Existe alguma dificuldade neste processo? ( ) sim ( ) não

Se sim, qual(is)?

( ) pinga fora da mucosa ocular ( ) pinga duas gotas de uma vez ( ) pinga no canto do olho

( ) Outra

Com qual freqüência você consulta com seu oftalmologista:
( ) Todo mês
( ) A cada três meses
( ) A cada seis meses
( ) Uma vez por ano 
Anexo - (Continuação)

Questionário - Dissertação de mestrado em Ciências da Saúde: Fatores para não-adesão à terapêutica medicamentosa do glaucoma

\section{Condições socioeconômicas:}

Renda: (De todas as pessoas que trabalham e moram na mesma residência)
( ) 300 reais
( ) 301 a 900 reais
( ) 1.501 a 2.100 reais
( ) 2.101 a 3.000 reais
( ) 901 a 1.500 reais
( ) maior que 3.000 reais

Quanto você gasta por mês para comprar os colírios?
( ) 1 a 20 reais
( ) 61 a 80 reais
( ) 21 a 40 reais
( ) mais que 80 reais
( ) 41 a 60 reais

Você deixa de usá-lo(s) caso seu dinheiro esteja acabando? ( ) sim ( ) não

Você recebe alguma ajuda para aquisição destes medicamentos e/ou doação dos mesmos? ( ) sim ( ) não

Se sim, de quem recebe esta ajuda e/ou doação?

Nível de escolaridade:
( ) $1^{\circ}$ Grau incompleto
( ) $2^{\circ}$ Grau completo
( ) $3^{\circ}$ Grau completo
( ) $1^{\circ}$ Grau completo
( ) $2^{\circ}$ Grau incompleto
( ) $3^{\circ}$ Grau incompleto
( ) Outra

\section{Doenças associadas:}

Você tem outra doença? ( ) sim ( ) não

Se sim, qual(is) seria $(\mathrm{m})$ esta(s) doença(s)? ( ) Diabetes ( ) Hipertensão ( ) Outra

Toma medicamentos para controlar a diabetes? ( ) sim ( ) não

Se sim, quais medicamentos e quantas vezes por dia ele(s) são tomados?

Medicamentos

( ) Metformina/Glucoformin ${ }^{\circledR}$

( ) Glibenclamida/Daonil ${ }^{\circledR}$

( ) Insulina/Biohulin ${ }^{\circledast}$

( ) Outro

№ de vezes que são tomados por dia

Toma medicamentos para controlar a Hipertensão? ( ) sim ( ) não

Se sim, quais medicamentos e quantas vezes por dia ele(s) são tomados?

Medicamentos

( ) Captopril/Capoten ${ }^{\circledast}$

( ) Hidroclorotiazida/Clorana ${ }^{\circledR}$

( ) 1 ( ) 2 ( ) 3

( ) $1($ ) $2($ ) 3

( ) $1($ ) $2($ ) 3

( ) $1($ ) $2($ ) 3

( ) Propranolol/Propranolol ${ }^{\circledR}$

( ) Atenolol/Atenol ${ }^{\circledR}$

( ) Furosemida/Lasix ${ }^{\circledast}$

( ) Enalapril/Renitec ${ }^{\circledR}$

( ) Clonidina/Atensina ${ }^{\circledR}$

( ) Nifedipina/Adalat ${ }^{\circledR}$

( ) Losartan/Cozaar ${ }^{\circledR}$

( ) Outro

№ de vezes que são tomados por dia

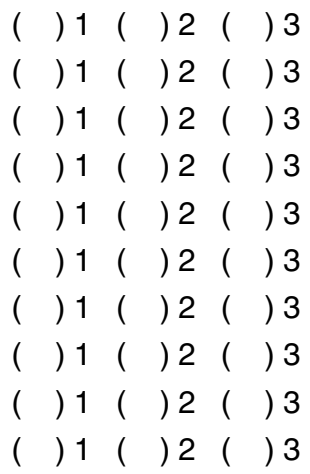

Nível de conhecimento da doença:

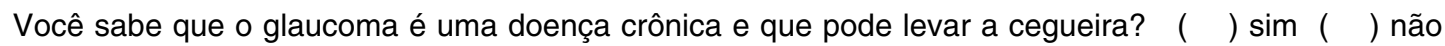

Você sabe que o tratamento do glaucoma é para vida toda e que os colírios devem ser usados constantemente para o glaucoma não evoluir? ( ) sim ( ) não 


\begin{tabular}{|lcc|}
\hline \multicolumn{3}{|c|}{ Tabela 1. Características demográficas dos pacientes } \\
Características & n (pacientes) & $\%$ \\
Sexo & & \\
Masculino & 28 & 27,5 \\
Feminino & 74 & 72,5 \\
Idade (média, desvio padrão e & $63,5 \pm 11,45$ anos & \\
amplitude) & $(34-86)$ & \\
Doses perdidas por semana & & \\
Nenhuma & 69 & 67,7 \\
Uma & 11 & 10,8 \\
Duas ou mais & 22 & 21,5 \\
Raça & & \\
Branca & 41 & 40,20 \\
Parda & 40 & 39,20 \\
Negra & 18 & 17,60 \\
Outra & 1 & 0,98 \\
Não informaram & 2 & 1,96 \\
Recebem ajuda para instilar os colírios & & \\
Sim & 24 & 23,5 \\
Não & 78 & 76,5 \\
Recebem ajuda para compra e/ou & & \\
doação de colírios & & \\
Sim & 16 & 15,7 \\
Não & 86 & 84,3 \\
\hline
\end{tabular}

\begin{tabular}{|lcr|}
\hline \multicolumn{3}{|c|}{ Tabela 2. Pacientes com hipertensão ou diabetes associados } \\
Doenças associadas & $\mathbf{n}$ (pacientes) & $\%$ \\
Hipertensão & 33 & 32,3 \\
Hipertensão e diabetes & 18 & 17,6 \\
Diabetes & 5 & 4,8 \\
\hline
\end{tabular}

Em relação ao fator sexo, os homens tiveram menor taxa de não-adesão quando comparados às mulheres, $21,7 \%$ contra $29,8 \%$ respectivamente. Porém, estes resultados não foram estatisticamente significativos $(\mathrm{p}=0,575)$. Alguns trabalhos ${ }^{(15-18,21-24)}$ indicaram a inexistência de associação significativa do sexo com a não-adesão ao tratamento. Contrariamente, um traba1 ho ${ }^{(3)}$ verificou maior taxa de não-adesão por parte dos homens, $37 \%$ contra $8 \%$, de forma significativa com $p<0,1$. Semelhantemente, outro estudo ${ }^{(20)}$ sugeriu que os homens tendem a perder mais doses de colírios, porém os dados levantados não foram estatisticamente significativos $(\mathrm{p}<0,24)$.

$\mathrm{O}$ fator idade não interviu de forma significativa com a nãoadesão. A faixa etária entre 50 e 59 anos, apresentou maior índice de não-adesão $(34,8 \%)$ quando comparada às outras três faixas etárias descritas no estudo. Outros trabalhos ${ }^{(15-18,21-25)}$ também demonstraram não haver interveniência da idade com a não-adesão ao tratamento medicamentoso do glaucoma.

Brancos e negros tiveram taxas de não-adesão bastante semelhantes, $24,4 \%$ e $22,2 \%$ respectivamente, enquanto os pardos tiveram a menor taxa de não-adesão com $15 \%$ de não aderentes. Não houve diferença estatisticamente significativa entre os grupos raciais descritos com a não-adesão $(p=0,559)$. Dois trabalhos ${ }^{(20,22)}$, apesar de definirem os grupos raciais de for-

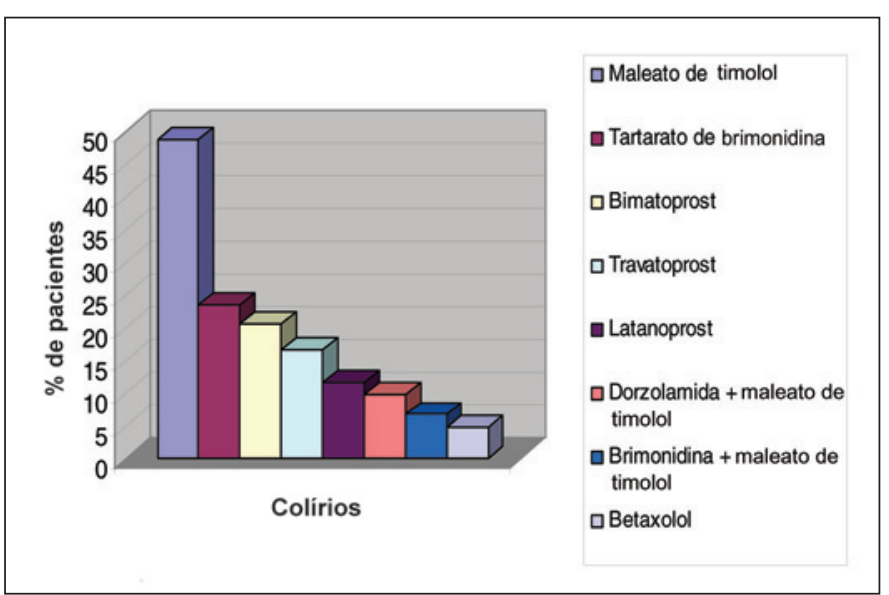

Figura 1 - Colírios mais utilizados pelo pacientes

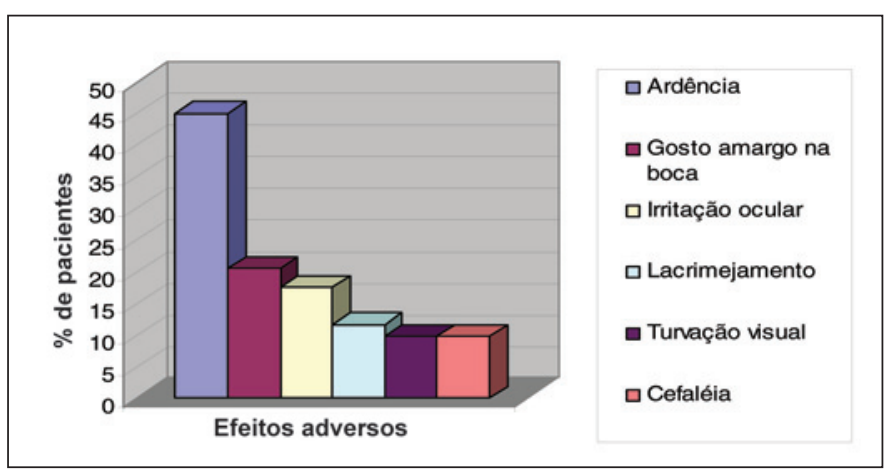

Figura 2 - Efeitos adversos mais notados pelos pacientes

mas diferentes ao nosso estudo, também não encontraram associação positiva entre raça e não-adesão. Outro trabalho(15) não encontrou diferenças significativas de não-adesão entre brancos e negros, porém quando estes foram comparados a um terceiro grupo racial denominado de outros, notou-se uma significativa menor adesão ao tratamento por parte deste último grupo.

Os efeitos adversos não foram considerados intervenientes a não-adesão $(\mathrm{p}=0,390)$. Outros estudos ${ }^{(14,17-18,20,26)}$ também apontaram para não interveniência a não-adesão. Em contrapartida, um estudo ${ }^{(3)}$ associou de forma significativa $(\mathrm{p}<0,1)$ a presença de efeitos adversos com a não-adesão.

Apesar disto, os efeitos adversos foram notados por pelo menos $45,1 \%$ dos pacientes. Em dois trabalhos ${ }^{(10,25)}$ as taxas de pacientes relatando sofrer com os efeitos adversos foram de $30 \%$ e $16 \%$ respectivamente.

Dois estudos $^{(8,27)}$ mostraram que $10 \%$ a $27 \%$ dos pacientes deixaram de usar os colírios devido aos efeitos adversos.

Semelhantemente a este estudo, dois outros ${ }^{(16-17)}$ verificaram que a presença de doenças coexistentes ao glaucoma, não intervieram de forma significativa com a não-adesão. Um estu$\mathrm{do}^{(3)}$ mostrou haver associação positiva com $\mathrm{p}<0,05$, sendo a artrite, a hipertensão, o diabetes e a aterosclerose, relatadas como principais doenças coexistentes. 


\begin{tabular}{|c|c|c|c|c|}
\hline Fatores & Aderentes & Não-aderentes & $\chi^{2}$ & $p$ valor \\
\hline \multicolumn{5}{|l|}{ Sexo } \\
\hline Masculino & 23 & 5 & 0,31 & $0,575 \#$ \\
\hline Feminino & 57 & 17 & $\mathrm{GL}=1$ & \\
\hline \multicolumn{5}{|l|}{ Faixa etária (em anos) } \\
\hline$<50$ & 31 & 4 & 6,78 & 0,079\# \\
\hline $50-59$ & 15 & 8 & $\mathrm{GL}=3$ & \\
\hline $60-69$ & 22 & 6 & & \\
\hline$>70$ & 32 & 4 & & \\
\hline \multicolumn{5}{|l|}{ Raça } \\
\hline Branca & 31 & 10 & 1,16 & 0,559\# \\
\hline Parda & 34 & 6 & $\mathrm{GL}=2$ & \\
\hline Negra & 14 & 4 & & \\
\hline \multicolumn{5}{|l|}{ Efeitos adversos } \\
\hline Sim & 61 & 19 & 1,04 & $0,390^{*}$ \\
\hline Não & 19 & 3 & $\mathrm{GL}=1$ & \\
\hline \multicolumn{5}{|l|}{ Doenças coexistentes } \\
\hline Sim & 61 & 18 & 0,04 & $0,849^{*}$ \\
\hline Não & 16 & 4 & $\mathrm{GL}=1$ & \\
\hline \multicolumn{5}{|l|}{ Cirurgia corretiva } \\
\hline Sim & 28 & 3 & 3,85 & 0,049\# \\
\hline Não & 51 & 19 & $\mathrm{GL}=2$ & \\
\hline \multicolumn{5}{|c|}{ Número máximo de aplicações de colírios por dia } \\
\hline 1 & 12 & 0 & 3,8 & $0,149 \#$ \\
\hline 2 & 64 & 21 & $\mathrm{GL}=2$ & \\
\hline 3 & 4 & 1 & & \\
\hline \multicolumn{5}{|l|}{ Número de colírios utilizados } \\
\hline 1 & 35 & 14 & 2,98 & 0,393\# \\
\hline 2 & 30 & 6 & $\mathrm{GL}=3$ & \\
\hline 3 & 14 & 2 & & \\
\hline 4 & 1 & 0 & & \\
\hline \multicolumn{5}{|l|}{ Dificuldade em pingar os colírios } \\
\hline Sim & 55 & 18 & 1,45 & $0,228 \#$ \\
\hline Não & 25 & 4 & $\mathrm{GL}=1$ & \\
\hline \multicolumn{5}{|l|}{ Nível de escolaridade } \\
\hline $1^{\circ}$ grau incompleto/completo & 49 & 13 & 3,10 & $0,377 \#$ \\
\hline $2^{\circ}$ grau incompleto/completo & 19 & 4 & $\mathrm{GL}=3$ & \\
\hline $3^{\circ}$ grau incompleto/completo & 6 & 4 & & \\
\hline Pós-graduação & 3 & 0 & & \\
\hline \multicolumn{5}{|l|}{ Renda familiar (em reais) } \\
\hline Até 900 & 39 & 9 & 0,58 & $0,748 \#$ \\
\hline 901 a 2100 & 26 & 9 & $\mathrm{GL}=2$ & \\
\hline$>2100$ & 14 & 4 & & \\
\hline \multicolumn{5}{|l|}{ Gasto mensal (em reais) } \\
\hline Até 40 & 29 & 12 & 3,24 & $0,198 \#$ \\
\hline 41 a 80 & 15 & 4 & $\mathrm{GL}=2$ & \\
\hline$>80$ & 34 & 5 & & \\
\hline \multicolumn{5}{|c|}{ Deixam de usar os colírios por falta de dinheiro } \\
\hline Sim & 10 & 7 & 4,89 & $0,035^{*}$ \\
\hline Não & 68 & 14 & $\mathrm{GL}=1$ & \\
\hline \multicolumn{5}{|l|}{ Consultas com oftalmologista } \\
\hline Uma vez por ano & 18 & 9 & 3,00 & 0,083\# \\
\hline Mais de uma consulta por ano & 62 & 13 & $\mathrm{GL}=1$ & \\
\hline$\# p=$ valor não corrigido; ${ }^{*} p=$ valor corri & & & & \\
\hline
\end{tabular}

A realização de cirurgias corretivas de glaucoma foi considerada como interveniente a não-adesão $(\mathrm{p}=0,049)$. $\mathrm{O}$ fato dos pacientes realizarem as cirurgias, pode tê-los induzidos a achar que estavam curados e conseqüentemente poderiam deixar de seguir a terapia medicamentosa. De forma contrária, um traba- lho ${ }^{(22)}$ não associou de forma positiva a não-adesão com a realização de cirurgias corretivas [OR=1,13; (IC 95\%=0,71-1,78)].

Todos os 22 pacientes considerados não aderentes, instilavam os colírios duas ou mais vezes ao dia. Situação concordante a outros estudos ${ }^{(13-15)}$, onde as taxas de não-adesão mostra- 
ram-se maiores em pacientes instilando colírios duas ou mais vezes ao dia. De forma similar, em dois trabalhos ${ }^{(17,26)}$ não foi verificada diferença significativa entre o número de instilações diárias e a não-adesão ( $\mathrm{p}=0,149)$.

Pacientes usando um único colírio tiveram maior índice de não-adesão $(28,7 \%)$ quando comparado àqueles utilizando dois ou mais colírios $(15,1 \%)$, porém não houve associação significativa com a não-adesão $(\mathrm{p}=0,393)$. Mesma situação foi encontrada em uma publicação americana ${ }^{(15)}$. Outros dois traba$\operatorname{lhos}^{(25-26)}$ não associaram de forma estatisticamente o número de colírios com a não-adesão. A adição de um segundo colírio à terapêutica, aumentou de forma estatisticamente significativa a taxa de não-adesão, conforme demonstrado em um artigo ${ }^{(1)}$.

Apesar do fator, dificuldade em pingar os colírios, não ter sido associado positivamente a não-adesão, o mesmo parece atrapalhar de várias maneiras a adesão à terapêutica medicamentosa. Dentre os 102 pacientes, 50 (49\%) relataram pingar os colírios fora da mucosa ocular, $25(24,5 \%)$, pingaram duas gotas por dose ao invés de uma e $24(23,5 \%)$ necessitaram da ajuda de outro indivíduo para instilar os colírios. A instilação de duas ou mais gotas por vez foi relatada por 37,6\% dos pacientes participantes de um estudo ${ }^{(11)}$ realizado nos Estados Unidos. A dependência de outra pessoa para aplicação dos colírios foi relatada por $21 \%$ dos pacientes envolvidos em um estudo britânico ${ }^{(12)}$. A inabilidade do paciente em acertar a gota de colírio na mucosa ocular ${ }^{(12,28-29)}$, além de gerar desperdício do medicamento, aumentando os custos do tratamento, contribui para uma dependência social do glaucomatoso com outro indivíduo. A aplicação de mais gotas de colírio não melhora o quadro clínico do glaucoma, e também torna o tratamento mais dispendioso, já que a mucosa ocular suporta no máximo uma gota de 20 microlitros ${ }^{(30)}$. Devido ao excesso de volume de colírio na mucosa ocular, o risco de efeitos adversos aumenta, ocorrendo absorção dos medicamentos via ducto nasolacrimal ${ }^{(31)}$. Neste estudo, $21(20,6 \%)$ pacientes relataram sentir gosto amargo na boca após utilização dos colírios, possibilitando a absorção de parte do conteúdo medicamentoso para a circulação sistêmica.

O nível de escolaridade dos pacientes foi relativamente baixo com $62(61 \%)$ pacientes com $1^{\circ}$ grau completo ou incompleto, contudo este fator não foi associado de forma significativa a não-adesão $(\mathrm{p}=0,377)$. Situação ainda pior foi mensurada em dois trabalhos ${ }^{(23-24)}$, com $38,7 \%$ e $67,6 \%$ respectivamente de pacientes analfabetos. Em ambos, o nível de escolaridade não foi associado de forma significativa com a não-adesão.

Dentre aqueles fatores de cunho econômico, renda familiar e gasto mensal com colírios não foram considerados fatores intervenientes a não-adesão, ao contrário do fator - deixar de usar os colírios por falta de dinheiro - que mostrou interveniência a não-adesão $(\mathrm{p}=0,035)$. Pode-se tentar explicar esta situação contraditória pelo fato dos pacientes receberem ajuda para compra dos colírios ou doações, em especial àquelas provenientes do Serviço Social do IPSEMG, onde pacientes com renda per capita comprovada de até um salário mínimo recebem os colírios gratuitamente. Este benefício foi possibi- litado a $6(5,8 \%)$ pacientes desta pesquisa. Outra explicação seria a baixa renda familiar de parte considerável dos pacientes (35\% recebem até $\mathrm{R} \$ 900,00$ mensais) e pelo alto custo dos colírios (33\% dos pacientes têm gasto mensal com colírios maior que $\mathrm{R} \$ 80,00)$. Pesquisadores brasileiros ${ }^{(32)}$ chegaram à conclusão de que pacientes os quais destinam maior proporção de sua renda ao tratamento, podem apresentar maior risco de baixa fidelidade ao seguimento medicamentoso. Verificaram ainda que, 45,2\% dos pacientes já haviam deixado de comprar colírios por falta de dinheiro.

Apesar de $27(26,5 \%)$ pacientes terem realizado apenas uma consulta ao ano com oftalmologista, o fator - consultas com oftalmologista - não foi associado de forma significativa a não-adesão. A melhora da adesão ao tratamento foi verificada com menores intervalos de tempo entre consultas ${ }^{(33)}$, fato não verificado neste estudo.

Esquecimento e instilação de colírios em horários inadequados, não foram avaliados em relação à associação dos mesmos com a não-adesão. O esquecimento do uso de colírios foi relatado por $11,7 \%$ dos pacientes, taxa bem inferior àquela encontrada em um estudo francês ${ }^{(16)}$, de 52,9\%. Dois traba$\operatorname{lhos}^{(20,25)}$ associaram de forma significativa o esquecimento com o não cumprimento à terapêutica medicamentosa. A instilação dos colírios em horários inadequados foi relatada por $10,7 \%$ dos pacientes. Outros quatro trabalhos ${ }^{(20,22-24)}$ mostraram haver associação positiva deste fator com a não-adesão.

\section{CONCLUSÃO}

Este estudo mostrou que mais de um quinto dos pacientes glaucomatosos não seguem o tratamento medicamentoso com colírios de forma adequada, situação contribuinte para o aumento da prevalência de casos de cegueira no Brasil.

Medidas como doação de colírios a todos os pacientes glaucomatosos de condições econômicas desfavoráveis e ações governamentais para diminuição dos preços dos colírios, especialmente daqueles mais caros (derivados das prostaglandinas), poderiam ajudar a diminuir as taxas de não-adesão ao tratamento com colírios antiglaucomatosos.

Além disso, informações a respeito da necessidade de continuidade do uso dos colírios antiglaucomatosos após a realização de cirurgias corretivas, poderiam ser salientadas aos pacientes, já que os mesmos tenderiam a achar que estão curados e a deixar de seguir a terapêutica medicamentosa.

\section{ABSTRACT}

Introduction: The treatment of glaucoma is made initially with the use of eyedrops, with the purpose to decrease intraocular pressure and to minimize the damages to the optic nerve. The lack of compliance with drug therapy can be influenced by factors related to patients, doctors and medicines. Purposes: To estimate the rate of noncompliance with the treatment with 
antiglaucoma eyedrops and to verify the intervenience of possible factors for noncompliance. Methods: A study of the transversal type was carried out, in the Ophthalmologic Clinic of the Hospital Governador Israel Pinheiro, in Belo Horizonte, Minas Gerais. Questionnaires were applied to 102 glaucoma patients to collect information about the level of noncompliance and the intervenience of possible factors for noncompliance with drug therapy. Results: The rate of noncompliance was $21.5 \%$. The factors: not using the eyedrops due to money shortage $(\mathrm{p}=0.035)$ and accomplishment of corrective surgery $(\mathrm{p}=0.049)$, significantly contributed to noncompliance. Conclusion: More than one fifth of the glaucoma patients does not follow the drug treatment in an adequate way. Donation of eyedrops to all the glaucoma patients without favorable economic conditions, governmental actions for the reduction of the prices of the eyedrops, information on the continuity of the use of the eyedrops after the accomplishment of corrective surgeries would help to decrease the rates of noncompliance.

Keywords: Glaucoma/drug therapy; Patient compliance; Treatment refusal; Ophthalmic solutions

\section{REFERÊNCIAS}

1. Robin AL, Covert D. Does adjunctive glaucoma therapy affect adherence to the initial primary therapy? Ophthalmology. 2005;112(5):863-8.

2. Granström PA. Glaucoma patients not compliant with their drug therapy: clinical and behavioural aspects. Br J Ophthalmol. 1982;66(7):464-70.

3. Bloch S, Rosenthal AR, Friedman L, Caldarolla P. Patient compliance in glaucoma. Br J Ophthalmol. 1977;61(8):531-4.

4. Coleman AL. Glaucoma. Lancet. 1999;354(9192):1803-10.

5. Silva MJL, Temporini ER, Neustein I, Araújo MEXS. Conhecimentos sobre prevenção e tratamento de glaucoma entre pacientes de unidade hospitalar. Arq Bras Oftalmol. 2004;67(5):785-90.

6. Tingey D, Bernard LM, Grima DT, Miller B, Lam A. Intraocular pressure control and persistence on treatment in glaucoma and ocular hypertension. Can J Ophthalmol. 2005;40(2):161-9.

7. Oliveira A, Paranhos Júnior A, Prata Júnior JA. Características dos pacientes atendidos pela primeira vez no Setor de Glaucoma da Universidade Federal de São Paulo - UNIFESP. Arq Bras Oftalmol. 2003;66(6):785-90.

8. Tsai JC, McClure CA, Ramos SE, Schlundt DG, Pichert JW. Compliance barriers in glaucoma: a systematic classification. J Glaucoma. 2003;12(5):393-8.

9. Nordstrom BL, Friedman DS, Mozaffari E, Quigley HA, Walker AM Persistence and adherence with topical glaucoma therapy. Am J Ophthalmol. 2005;140(4):598-606.

10. Deokule S, Sadiq S, Shah S. Chronic open angle glaucoma: patient awareness of the nature of the disease, topical medication, compliance and the prevalence of systemic symptoms. Ophthalmic Physiol Opt. 2004;24(1):9-15.
11. Kass MA, Hodapp E, Gordon M, Kolker AE, Goldberg I. Part I. Patient administration of eyedrops: interview. Ann Ophthalmol. 1982;14(8):775-9.

12. Winfield AJ, Jessiman D, Williams A, Esakowitz L. A study of the causes of noncompliance by patients prescribed eyedrops. Br J Ophthalmol. 1990; 74(8):477-80.

13. Kass MA, Meltzer DW, Gordon M, Cooper D, Goldberg J. Compliance with topical pilocarpine treatment. Am J Ophthalmol. 1986;101(5):515-23.

14. Kass MA, Gordon M, Morley RE Jr, Meltzer DW, Goldberg JJ. Compliance with topical timolol treatment. Am J Ophthalmol. 1987;103(2):188-93.

15. Gurwitz JH, Glynn RJ, Monane M, Everitt DE, Gilden D, Smith N, Avorn J. Treatment for glaucoma: adherence by the elderly. Am J Public Health. 1993; 83(5):711-6.

16. Bour T, Blanchard F, Segal A. [Therapeutic observance and life of patients with primary open-angle glaucoma. Apropos of 341 cases in the department of Marne]. J Fr Ophtalmol. 1993;16(6-7):380-91. French.

17. Gurwitz JH, Yeomans SM, Glynn RJ, Lewis BE, Levin R, Avorn J. Patient noncompliance in the managed care setting. The case of medical therapy for glaucoma. Med Care. 1998;36(3):357-69.

18. Rotchford AP, Murphy KM. Compliance with timolol treatment in glaucoma. Eye. 1998;12(Pt 2):234-6.

19. Stewart WC, Konstas AG, Pfeiffer N. Patient and ophthalmologist attitudes concerning compliance and dosing in glaucoma treatment. J Ocul Pharmacol Ther. 2004;20(6):461-9.

20. Patel SC, Spaeth GL. Compliance in patients prescribed eyedrops for glaucoma. Ophthalmic Surg. 1995;26(3):233-6.

21. Rocheblave A. [Cooperation of patients with chronic primary open-angle glaucoma]. J Fr Ophtalmol. 1983;6(10):837-41. French.

22. Kosoko O, Quigley HA, Vitale S, Enger C, Kerrigan L, Tielsch JM. Risk factors for noncompliance with glaucoma follow-up visits in a residents' eye clinic. Ophthalmology. 1998;105(11):2105-11.

23. Wane AM, Ndiaye MR, Wade A, Ndiaye PA, Ba EA, de Medeiros ME, et al. [Compliance of medical treatment in primitive glaucoma open angle]. J Fr Ophtalmol. 2003;26(10):1039-44. French.

24. Khandekar R, Shama Mel-S, Mohammed AJ. Noncompliance with medical treatment among glaucoma patients in Oman - a cross-sectional descriptive study. Ophthalmic Epidemiol. 2005;12(5):303-9.

25. Sleath B, Robin AL, Covert D, Byrd JE, Tudor G, Svarstad B. Patientreported behavior and problems in using glaucoma medications. Ophthalmology. 2006;113(3):431-6.

26. Granström PA, Norell S. Visual ability and drug regimen: relation to compliance with glaucoma therapy. Acta Ophthalmol (Copenh). 1983;61(2):206-19.

27. Taylor SA, Galbraith SM, Mills RP. Causes of non-compliance with drug regimens in glaucoma patients: a qualitative study. J Ocul Pharmacol Ther. 2002;18(5):401-9.

28. Busche S, Gramer E. [Improved eyedrop administration and compliance in glaucoma patients. A clinical study]. Klin Monatsbl Augenheilkd. 1997; 211(4):257-62. German.

29. Salyani A, Birt C. Evaluation of an eye drop guide to aid self-administration by patients experienced with topical use of glaucoma medication. Can J Ophthalmol. 2005;40(2):170-4.

30. Stillitano IG, Lima MG, Ribeiro MP, Cabral J, Brandt CT. Impacto econômico do custo de colírios no tratamento do glaucoma. Arq Bras Oftalmol. 2005;68(1):79-84.

31. Hosoda M, Yamabayashi S, Furuta M, Tsukahara S. Do glaucoma patients use eye drops correctly? J Glaucoma. 1995;4(3):202-6.

32. Silva LMS, Vasconcellos JPC, Temporini ER, Costa VP, Kara-José N. Tratamento clínico do glaucoma em um hospital universitário: custo mensal e impacto na renda familiar. Arq Bras Oftalmol. 2002;65(3):299-303.

33. Norell SE. Monitoring compliance with pilocarpine therapy. Am J Ophthalmol. 1981;92(5):727-31. 